\title{
The difficulty of the postural control task affects multi-muscle control during quiet standing
}

\author{
X. García-Massó ${ }^{1} \cdot$ M. Pellicer-Chenoll ${ }^{2}$ L. M. Gonzalez ${ }^{2} \cdot$ J. L. Toca-Herrera ${ }^{3}$
}

Received: 13 September 2015 / Accepted: 18 February 2016 / Published online: 4 March 2016

(C) The Author(s) 2016. This article is published with open access at Springerlink.com

\begin{abstract}
The aim of this study was to compare the electromyographic (EMG) coherence between the lower limb and the core muscles when carrying out two postural tasks at different difficulty levels. EMG was recorded in 20 healthy male subjects while performing two independent quiet standing tasks. The first one involved a bipedal stance with the eyes open, while the second consisted of a dominant unipedal stance also with the eyes open. The obtained EMG signals were analysed by computing estimations of EMG-EMG coherence between muscle pairs, both singly (single-pair estimations) and combined (pooled estimations). Pooled and single coherence of anterior, posterior, core, antagonist and mixed pairs of muscles were significant in the $0-5 \mathrm{~Hz}$ frequency band. The results indicate that core and antagonist muscle groups, such as the anterior and posterior muscles, share low-frequency neural inputs $(0-5 \mathrm{~Hz})$ which could be responsible of the M-modes assembly. The core muscles could therefore provide the necessary synergy to maintain spine stability during the balancing exercise. Finally, differences in EMG-EMG coherence suggest that the muscle synergies formed during
\end{abstract}

Electronic supplementary material The online version of this article (doi:10.1007/s00221-016-4602-z) contains supplementary material, which is available to authorized users.

J. L. Toca-Herrera

jose.toca-herrera@boku.ac.at

1 Department of Teaching Music, Arts and Body Expression, University of Valencia, Valencia, Spain

2 Department of Physical Education and Sport, University of Valencia, Valencia, Spain

3 Department of Nanobiotechnology, Institute for Biophysics, University of Natural Resources and Life Sciences Vienna (BOKU), Muthgasse 11, 1190 Vienna, Austria unipedal stance tasks are different from those established during bipedal stance.

Keywords Electromyography $\cdot$ Coherence $\cdot$ Synergy . Quiet standing

\section{Introduction}

From a mechanical point of view, human vertical posture is an unstable system that needs control mechanisms to maintain the centre of gravity inside the support base (Suzuki et al. 2012). Even though several studies have focused on modelling quiet stance control obtaining important information about its mechanism, the system of study was reduced to either an inverted or a double-inverted pendulum (Masani et al. 2003; Asai et al. 2009; Gawthrop et al. 2011; Suzuki et al. 2012). However, experimental data show that a great number of joints and muscles are involved in quiet standing balance, resulting in redundant degrees of freedom (Günther et al. 2009, 2011). Bernstein (1967) postulated that the central nervous system (CNS) simplifies the redundant degrees of freedom by activating synergies (Shumway-Cook and Woollacott 2007). These synergies are formed by groups of muscles that act together to perform the same function.

On this question, Krishnamoorthy et al. (2003) determined the existence of three synergistic muscle groups (known as muscle modes or M-modes) that maintain the balance in an upright stance using the uncontrolled manifold (UCM) hypothesis. The first group is composed by the muscles of the posterior area (e.g. gastrocnemius and biceps femoris), the second one by the anterior muscles (e.g. tibialis anterior and rectus femoris) and the last one by the rectus abdominis. This clustering was obtained taking 
into account the effects of these muscles in CoP displacement: the anterior muscles are used to move $\mathrm{CoP}$ forwards, while the posterior muscles displace it backwards. Another study found M-modes co-contraction during load release tasks in unstable upright posture (Krishnamoorthy et al. 2004). These M-modes were presented at calf (tibialis anterior and triceps surae), thigh (vastus lateralis, rectus femoris and biceps femoris) and core muscles (rectus abdominis and erector spinae), and appeared as a consequence of the high task difficulty (Krishnamoorthy et al. 2004). Overall, in this study the authors determined the existence of five different M-modes (i.e. two reciprocal and three co-contractions). Moreover, the authors supported the hypothesis that the CNS chooses only three of these M-modes to control the posture during upright stance. This choice can depend of factors such as task difficulty or expertise.

Furthermore, it was demonstrated that anterior and posterior M-modes are correlated neuronal inputs (DannaDos-Santos et al. 2014, 2015). This was figured out from electromyographic (EMG) coherence analysis between the muscles that form the M-modes during bipedal quiet stance. Thus, the mechanism responsible for the synergic action could be attributed to these correlated inputs that the CNS sends to the muscles.

The effect of visual information in the EMG-EMG coherence of anterior and posterior M-modes has also been studied (Danna-Dos-Santos et al. 2015). The authors observed that visual information plays a key role in the generation of common inputs to the muscles. In the same work, the authors postulated that it might be relevant to perform studies taking into account more muscle groups and conditions, in order to gain understanding of the CNS role and muscle synergies in balance control.

Moreover, during daily and sport activities single-leg stance periods are performed. This postural configuration has been considered to be more complex than bipedal stance from the point of view of postural control (Vuillerme et al. 2001; Paillard et al. 2006). In addition, several studies have focused on the effect of training (Paillard et al. 2006), fatigue (Bizid et al. 2009; Bisson et al. 2011) or foot type (Hertel et al. 2002) in unipedal stability. Nevertheless, there are few studies that confirm muscle coordination or muscle synergies during unipedal stance test. Danna-dos-Santos and co-workers found the anterior and posterior M-modes during unipedal stance task in which voluntary sway in anteroposterior direction was performed (Danna-Dos-Santos et al. 2008). Nevertheless, M-modes co-contraction was not found although the task difficulty.

The primary aim of the present study was to analyse the EMG-EMG coherence between the muscles that forms reciprocal (i.e. anterior and posterior) and co-contraction (i.e. at calf, thigh and core) M-modes. Our second aim was to determine the effect of the task difficulty on muscle coherence by comparing EMG-EMG coherence between bipedal and unipedal stance conditions.

\section{Methods}

\section{Participants}

Prior to subject's recruitment, a sample size calculation was performed using $G^{*}$ Power 3.1 (University of Düsseldorf, Düsseldorf, Germany). Based on the data published in Danna-Dos-Santos et al. (2015), a sample size of 16 was necessary to detect an effect size of 0.92 when Wilcoxon signed-rank test was performed. This effect size was obtained during comparisons between bipedal eyes open and bipedal eyes closed conditions. We assume that effect size equal or higher than 0.92 will be found between bipedal and unipedal conditions. Therefore, 20 subjects were recruited to participate in the study (four more due to possible loss of data).

The average (SD) age, weight and height were 23.3 (4.52) years, $78.2(9.84) \mathrm{kg}$ and $1.78(0.07) \mathrm{m}$, respectively. The participants had no history of neurological or muscular disorders. Eighteen subjects were right-footed and two leftfooted, based on their kicking preference. Informed consent was obtained from all individual participants included in the study. The research project was approved by the University Institutional Review Board, and the procedures were in accordance with the Declaration of Helsinki.

\section{Experimental procedure}

All the participants performed two independent quiet standing trials. One involved a bipedal stance with the eyes open, and the other consisted of a dominant unipedal stance, also with the eyes open (Fig. 1). The order was randomized to avoid any influence of this factor on the results. Each trial had duration of $50 \mathrm{~s}$ with a rest period of $60 \mathrm{~s}$ between trials. In the bipedal stance, the subjects were barefoot and still in a relaxed manner with their arms by their sides. Each subject adopted the same foot placement (i.e. heels separated by the width of the shoulders and toes pointing forward). In the unipedal stance, the subjects stood barefoot on their dominant foot. The knee of the non-supporting leg was flexed $90^{\circ}$ to ensure that it did not make contact with the floor during the entire trial. If the subjects bent their trunk or arms, as well as they touched down with the nonsupporting limb, the trial was discarded. Therefore, after a recovery period of $60 \mathrm{~s}$ the participants performed another trial. This procedure was repeated until one successful trial could be recorded. A point of reference $(5 \mathrm{~cm}$ in diameter) was placed in front of the subject at eye level at a distance of $2 \mathrm{~m}$ for each trial. All the subjects were informed of the 
Fig. 1 Experimental conditions and examples of tibialis anterior electromyography and centre of pressures signal. In a, the centre of pressure displacement and the electromyographic activity of tibialis anterior during bipedal stance condition are shown. $U p$, the centre of pressure displacement in anteroposterior (AP; in black) and mediolateral (ML; in grey) is indicated. Down, the raw electromyographic signal of tibialis anterior is plotted in grey, while the root mean square is plotted in black. In $\mathbf{b}$, the centre of pressure displacement and the electromyographic activity of tibialis anterior during unipedal stance condition are shown. $U p$, the centre of pressure displacement for anteroposterior (AP; in black) and mediolateral (ML; in grey) is shown. Down, the raw electromyographic signal of tibialis anterior is plotted in grey, while the root mean square is marked in black
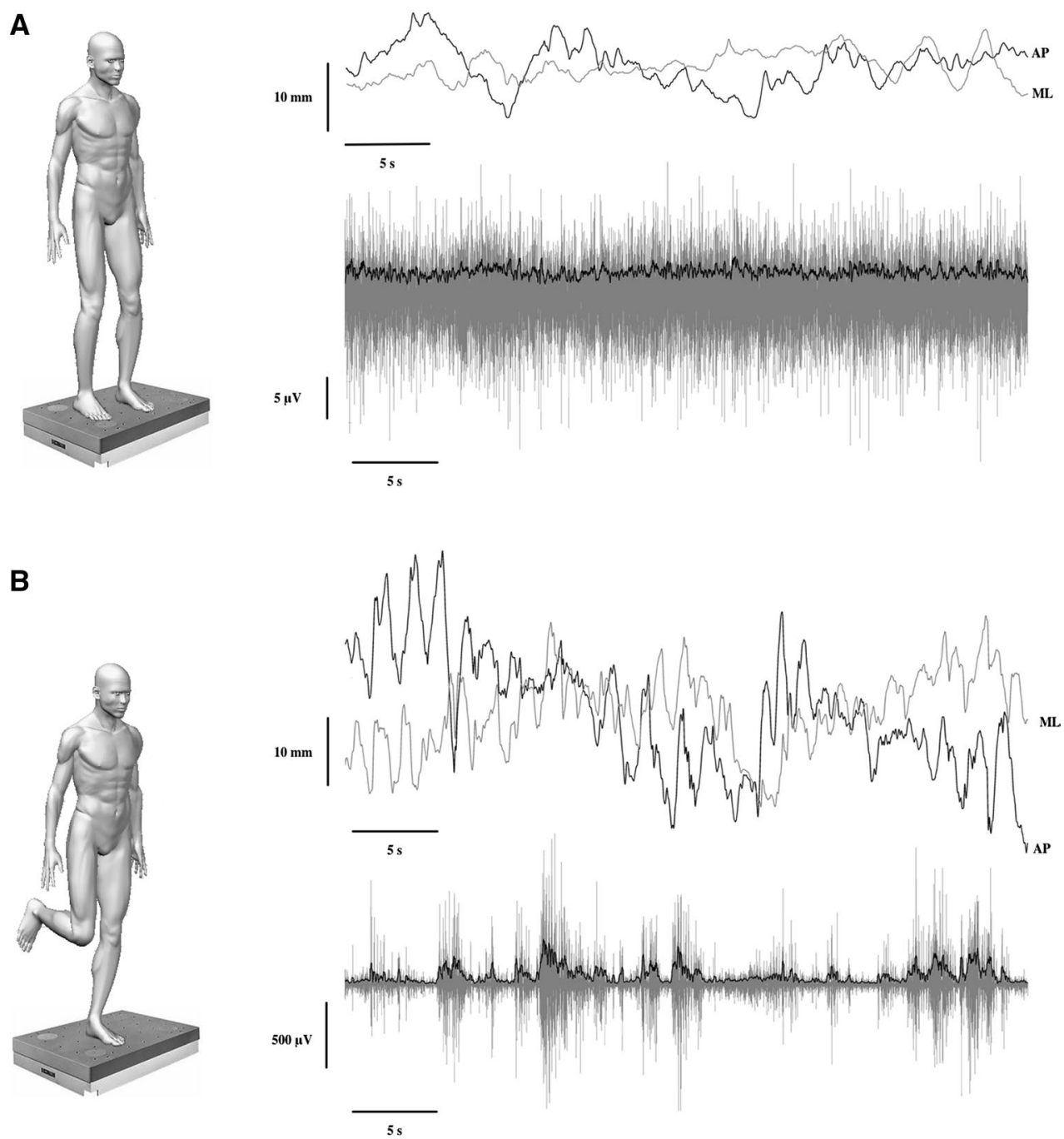

importance of maintaining these postures and were asked to stand as still as possible.

\section{Centre of pressure measurements}

A $400 \mathrm{~mm} \times 600 \mathrm{~mm} \times 45 \mathrm{~mm}$ portable force plate (9253B11, Kistler Instrument AG, Winterthur, Switzerland) was used to acquire the centre of pressure displacements. The platform was placed on a stable surface on the floor to avoid distortion and noise in the signal. The force plate contained four piezoelectric sensors and each recorded the force produced in three spatial directions. The forces that exerted along the $x-, y$ - and $z$-axis were recorded at a frequency of $1 \mathrm{kHz}$, amplified (Kistler amplifier) and converted A/D using a 16-bit card (CIODAS 1600). The centre of pressure $(\mathrm{CoP})$ displacement in anteroposterior $(\mathrm{AP})$ and mediolateral (ML) directions was obtained using the manufacturer's analysis software (Bioware, Kistler Instrument AG, Winterthur, Switzerland).

\section{Electromyography measurements}

To acquire the surface electromyographic signals produced during the trials, we used a ME6000 biomonitor (Mega Electronics, Ltd., Kuopio, Finland). Prior placing the electrodes, the skin was prepared by shaving the area and also cleaning it with alcohol in order to reduce impedance as much as possible. Pre-gelled bipolar surface electrodes Ag/ $\mathrm{AgCl}$ (Blue sensor M-00-S; Medicotest, Ølstykke, Denmark) were placed at an interelectrode distance of $20 \mathrm{~mm}$ on the following muscle groups: 1. gastrocnemius medialis (GM), 2. tibialis anterior (TA), 3. vastus medialis (VM), 4. biceps femoris (BF), 5. rectus abdominis (RA), 6. external oblique (EO) and 7. erector spinae (ES). The reference electrode was placed between the active electrodes at approximately $10 \mathrm{~cm}$ from each one, as per the manufacturer's specifications. The electrodes were placed according to the SENIAM recommendations (www.seniam.org) on the dominant side of the body. 
All signals were acquired at a sampling frequency of $1 \mathrm{kHz}$, amplified and converted analogue/digital. All records of myoelectrical activity $(\mu \mathrm{V})$ were stored on hard drive for later analysis. A trigger was used to synchronize CoP and EMG measurements.

\section{Data processing}

The data analysis was performed with MATLAB R2013a (Mathworks Inc, Natick, USA). CoP signals were low-pass dual-pass filtered using a second-order Butterworth IIR filter with a cut-off frequency of $15 \mathrm{~Hz}$. The first $10 \mathrm{~s}$ were then cut out before the computation of the variables. In the time domain, the balance variables computed were the $95 \%$ confidence ellipse area (EA), mean velocity (MV) in anteroposterior (AP) and mediolateral (ML) direction and root mean square (RMS) amplitude in AP and ML direction. Frequency domain analysis was also performed. The power spectral density was estimated using a fast Fourier transformation (periodogram function in MATLAB) using a Hanning window of $2^{10}$ data points. Finally, the median frequency and the $80 \%$ energy frequency ( $80 \%$ of spectral energy is below this frequency) were calculated.

EMG signals were pre-processed to delete noise and interference. First, we applied a band-pass second-order Butterworth IIR filter with 20-400 cut-off frequencies in direct and reverse directions. Independent component analysis was used to cancel out electrocardiogram interference in the trunk muscles (i.e. RA, ES and EO) (Mak et al. 2010). This was of great importance because the presence of electrocardiogram signals in EMG could increase the coherence level between core muscles (Grosse et al. 2002).

The first $10 \mathrm{~s}$ of the signals were discarded in order to use the same time period in the CoP and EMG signals. In the time domain, a RMS moving window of $100 \mathrm{~ms}$ was applied. The mean value of the RMS signal was then computed as a variable of the magnitude of muscle activation.

In the frequency domain, EMG signals were analysed by estimating the EMG-EMG coherence between muscle pairs (single-pair estimations) separately and combined (pooled estimations). Coherence analysis was performed using the full-wave rectified EMG signal. Rectification was performed following the recommendations established in computational studies (Boonstra and Breakspear 2012; Farina et al. 2013; Ward et al. 2013) as well as previous studies on intermuscular coherence during quiet standing (Danna-Dos-Santos et al. 2014, 2015; Obata et al. 2014).

The procedure used for the EMG-EMG coherence analysis was similar to those reported previously (Poston et al. 2010; Danna-Dos-Santos et al. 2015). The functional synergies related to the anterior and posterior M-modes described above were tested in this study (Table 1). Common neural inputs in the core muscles were also established. Moreover,
Table 1 Muscle pairs used during intermuscular coherence analyses (pooled and single pair)

\begin{tabular}{lll}
\hline & Muscle pair & Muscular synergy \\
\hline 1 & GM-BF & Posterior \\
2 & GM-ES & Posterior \\
3 & BF-ES & Posterior \\
4 & TA-VM & Anterior \\
5 & TA-RA & Anterior \\
6 & VM-RA & Anterior \\
7 & RA-ES & Core \\
8 & RA-EO & Core \\
9 & ES-EO & Core \\
10 & GM-VM & Mixed \\
11 & GM-RA & Mixed \\
12 & VM-ES & Mixed \\
13 & TA-BF & Mixed \\
14 & TA-ES & Mixed \\
15 & BF-RA & Mixed \\
16 & TA-GM & Antagonist \\
17 & VM-BF & Antagonist \\
\hline
\end{tabular}

$T A$ tibialis anterior, $G M$ gastrocnemius medialis, $V M$ vastus medialis, $B F$ biceps femoris, $R A$ rectus abdominis, $E O$ external oblique, $E S$ erector spinae

antagonist synergies of calf and thigh muscles were calculated. Finally, coherence was computed between muscles with no synergistic relation (mixed group in Table 1). The latter muscle pair group performed the role of a control group. Including all these muscle pairs thus allowed us to determine the existence of: (1) correlated neural commands in the anterior muscles, (2) correlated neural commands in the posterior muscles, (3) correlated neural commands in the core muscles and (4) correlated neural commands in antagonist leg muscles.

Single-pair coherence estimations were performed by dividing the squared cross-spectrum of two EMG signals by the product of the auto-spectrum of each signal:

$\left|R_{x y}(\lambda)\right|^{2}=\frac{\left|f_{x y}(\lambda)\right|^{2}}{\left|f_{x x}(\lambda) f_{y y}(\lambda)\right|}$

where $R_{x y}(\lambda)$ is the intermuscular coherence, $f_{x y}(\lambda)$ is the cross-spectrum between both EMG signals, $f_{x x}(\lambda)$ is the auto-spectrum of the first EMG signal and $f_{y y}(\lambda)$ is the auto-spectrum of the second EMG signal.

The estimated coherence was obtained using the Welch method with a non-overlapping Hanning window of 1024 points (frequency resolution $=0.98 \mathrm{~Hz}$ ). The range of the frequencies analysed in this study varied from 0 to $55 \mathrm{~Hz}$. The coherence confidence limit was set at 0.0739 , following the equation proposed by Rosenberg et al. (1989). 
Table 2 Differences between conditions in $\mathrm{CoP}$ variables

\begin{tabular}{llcclrr}
\hline & Direction & Bipedal & Unipedal & $z$ & $r$ & $p$ value \\
\hline EA $\left(\mathrm{mm}^{2}\right)$ & - & $114.60(170.09)$ & $628.23(307.99)$ & -3.88 & -0.61 & $<0.001$ \\
RMS $(\mathrm{mm})$ & ML & $2.19(2.10)$ & $5.35(1.15)$ & -3.92 & -0.62 & $<0.001$ \\
& AP & $3.46(2.19)$ & $6.62(2.49)$ & -2.84 & -0.45 & 0.005 \\
MV $\left(\mathrm{mm} \mathrm{s}^{-1}\right)$ & ML & $3.53(2.12)$ & $24.52(17.01)$ & -3.92 & -0.62 & $<0.001$ \\
& AP & $5.02(2.35)$ & $22.74(12.88)$ & -3.92 & -0.62 & $<0.001$ \\
F80 (Hz) & ML & $0.80(0.41)$ & $1.12(0.49)$ & -3.3 & -0.52 & 0.001 \\
& AP & $0.59(0.47)$ & $1.05(0.41)$ & -3.92 & -0.62 & $<0.001$ \\
F50 (Hz) & ML & $0.54(0.21)$ & $0.67(0.30)$ & -2.24 & -0.35 & 0.025 \\
& AP & $0.32(0.16)$ & $0.67(0.34)$ & -3.87 & -0.61 & $<0.001$ \\
\hline
\end{tabular}

Data are expressed as median (interquartile range)

$E A$ ellipse area, $R M S$ root mean square, $M V$ mean velocity, $F 8080 \%$ energy frequency, $F 5050 \%$ energy frequency, $A P$ anteroposterior, $M L$ mediolateral
Coherence estimations were normalized (Fisher transformation) to perform comparisons between the two experimental conditions. The coherence signal was an integer over the range interval between 0 and $5 \mathrm{~Hz}$ (i.e. common drive) because significant coherence was found only in that frequency band (Poston et al. 2010).

Five pooled coherence estimations were also quantified. The first included the three pairs of anterior muscles (TA/ $\mathrm{VM}, \mathrm{TA} / \mathrm{RA}$ and VM/RA), the second the pairs of posterior muscles (GM/BF, GM/ES and BF/ES), the third the pairs of core muscles (RA/ES, RA/EO and ES/EO), the fourth the mixed pairs (Table 1) and the last the antagonist pairs (TA/GM and VM/BF). The pooled coherence was computed as the weighted average of individual coherence estimation pairs (Poston et al. 2010; Danna-Dos-Santos et al. 2014, 2015; Obata et al. 2014). The pooled coherence was normalized using Fisher transformation and integer in the same frequency interval as single coherence.

\section{Statistical analysis}

Statistical analysis was performed with SPSS 20 (IBM, Armonk, USA). The normality (Shapiro-Wilk test) assumption was checked, and as several variables did not meet this assumption, nonparametric tests were applied (supplementary material). Descriptive methods were used to compute the median and the interquartile range. Wilcoxon signed-rank tests were applied to establish differences between conditions (i.e. bipedal and unipedal) in $\mathrm{CoP}$ variables and mean RMS electromyography. Friedman's ANOVA was applied to establish differences between groups of muscle pairs (i.e. anterior, posterior, core, mixed and antagonist groups) as regards pooled coherence integral values. Friedman's ANOVA was applied to establish differences in the single coherence between pairs of muscles that form the same synergy. The follow-up to Friedman's ANOVA was carried out by means of multiple
Wilcoxon signed-rank tests. Due to the multiple comparisons, a Bonferroni correction was used. Finally, differences in the pooled and single coherence between conditions (i.e. bipedal and unipedal) were established by means of the Wilcoxon signed-rank test. The level of significance was set at $p=0.05$.

\section{Results}

\section{CoP variables}

Significant differences in all the $\mathrm{CoP}$ variables were found between bipedal and unipedal stance conditions. In fact, all the variables presented a higher median value in the unipedal than the bipedal stance condition. Table 2 shows the descriptive statistics as well as Wilcoxon signed-rank test statistic, effect size and $p$ values.

\section{EMG time domain variables}

Significant differences in the mean RMS between conditions were observed (Fig. 2). The mean RMS was higher in the unipedal stance condition in TA $(z=-3.92 ; p<0.001$; $r=-0.62)$, GM $(z=-3.92 ; p<0.001 ; r=-0.62)$, $\mathrm{VM}(z=-3.66 ; p<0.001 ; r=-0.58)$, EO $(z=-2.54$; $p=0.01 ; r=-0.4)$ and $\mathrm{ES}(z=-3.21 ; p=0.001$; $z=0.51)$.

\section{Pooled coherence}

As the pooled coherence of anterior, posterior, core, mixed pairs of muscles and antagonist muscles was significant in the $0-5 \mathrm{~Hz}$ frequency band (Fig. 3), the integral value of the frequency bands was compared between these muscles groups as well as between the conditions. It is important to note that the coherence of core muscles reached 


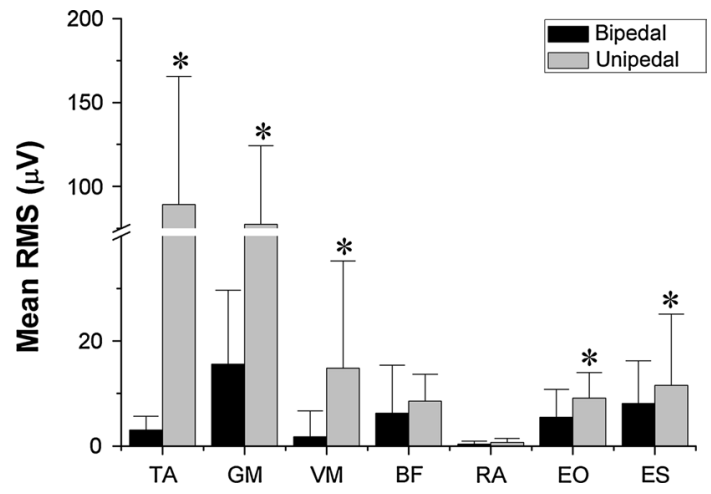

Fig. 2 Mean electromyography RMS comparison between different conditions. Bars represent the median value and error bars the interquartile range. Asterisk indicates significant differences related to bipedal condition $(p<0.05)$. TA tibialis anterior, $G M$ gastrocnemius medialis, $V M$ vastus medialis, $B F$ biceps femoris, $R A$ rectus abdominis, $E O$ external oblique, $E S$ erector spinae

the significance level throughout almost all the frequency range.

Friedman's ANOVA revealed a significant effect of the group on the common drive band $\left(\chi_{4}^{2}=23.88 ; p<0.001\right)$ in the bipedal stance condition. The pairwise comparisons are shown in Fig. 4. In this band, the mixed group showed a lower integral than the posterior, core and antagonist groups.

In the unipedal stance, Friedman's ANOVA showed an effect of the muscle group in common drive $\left(\chi_{4}^{2}=54.2\right.$; $p<0.001)$. In this condition, the mixed and anterior groups showed lower values than the posterior, core and antagonist muscle groups (Fig. 4). Moreover, the antagonist integral value was larger than the integral values of core and posterior groups.

A comparison of the conditions (Fig. 4) shows that the mixed group integral value was higher in unipedal than in bipedal stance $(z=-3.43 ; p=0.001 ; r=-0.54)$. Finally, the posterior muscle group integral value was higher in bipedal than in unipedal stance in the common drive frequency band $(z=-2.35 ; p=0.019 ; r=-0.37)$.

\section{Single coherence}

As the coherence of single muscle pairs reached values above the threshold confidence level in the $0-5 \mathrm{~Hz}$ frequency band, the integral values were computed in the same way as the pooled coherence values.

No differences between the integral values of the pairs of muscles involved in the same M-mode in the bipedal stance were found. Nevertheless, significant differences between
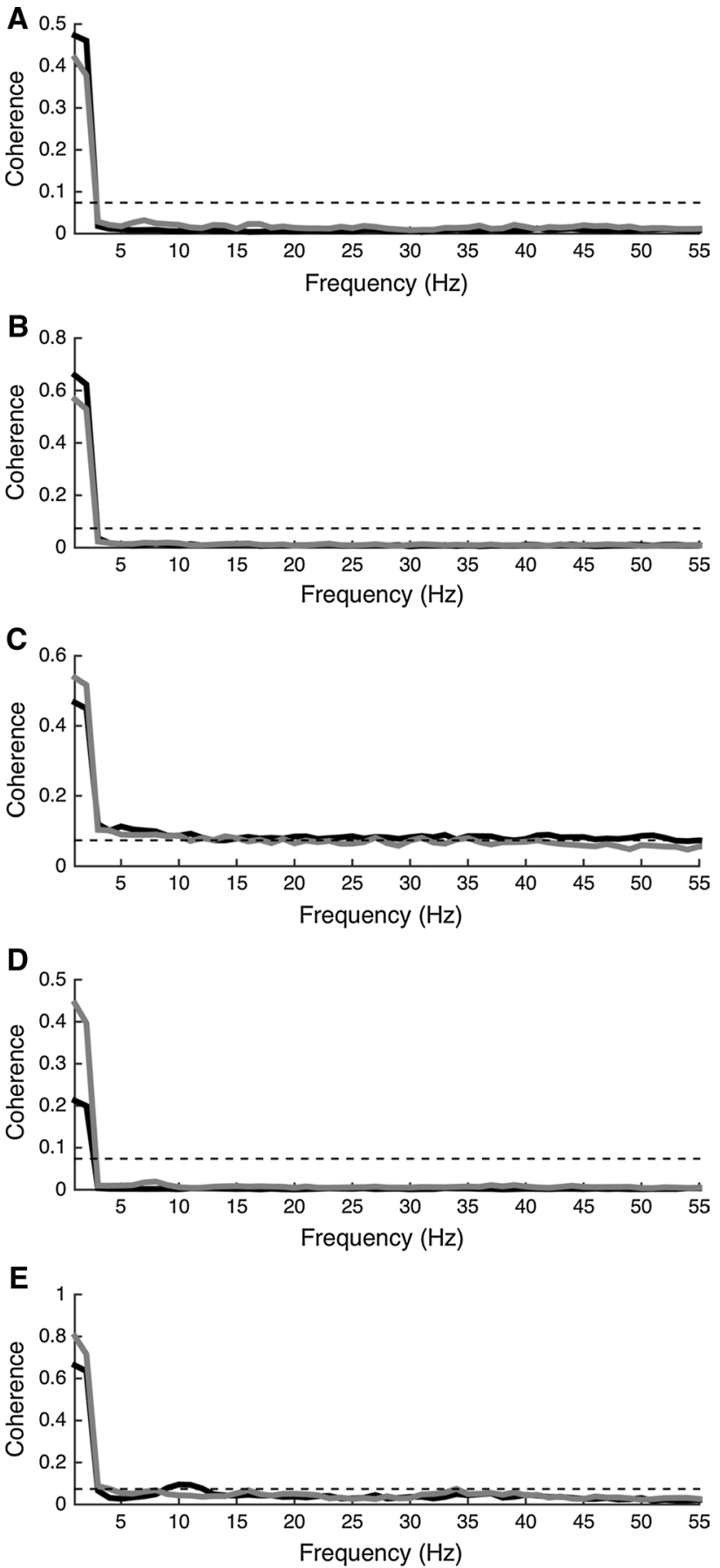

Fig. 3 EMG-EMG pooled coherence. a Pooled coherence for anterior M-mode muscles. b Pooled coherence for posterior M-mode muscles. c Pooled coherence for core muscles. d Pooled coherence for mixed muscles group. e Pooled coherence for the antagonist muscle group. The coherence of bipedal stance condition is marked in black, while the dark grey line relates to the coherence during unipedal stance condition. The dotted line represents the level of significance for coherence analysis (i.e. 0.0739) 


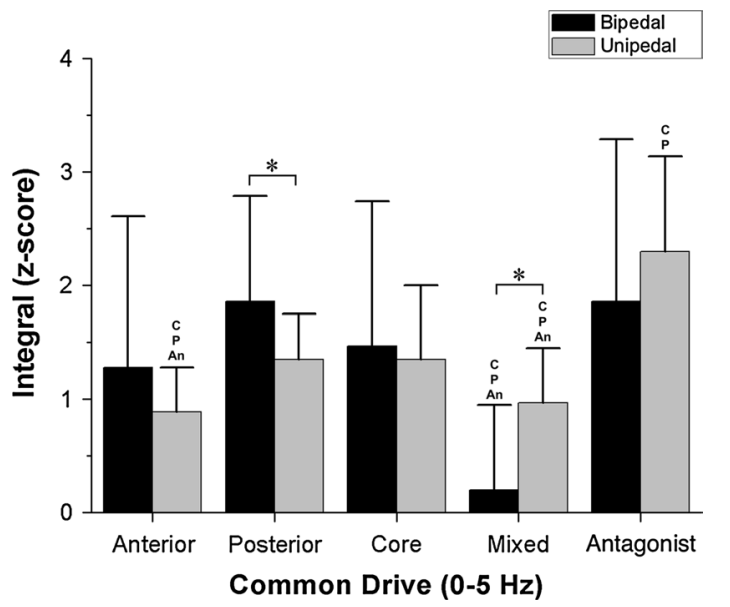

Fig. 4 Comparison between muscle groups and conditions versus the integral of the normalized coherence between 0 and $5 \mathrm{~Hz}$. The bars represent the median and the error bars the interquartile range. Asterisk indicates the significant differences between bipedal and unipedal conditions. An refers to significant differences related to the antagonist M-mode. $P$ indicates significant differences related to posterior M-mode. $C$ relates to significant differences of the core muscles

TA/GM and VM/BF integral value in bipedal condition $(z=-2.58 ; p=0,01 ; r=0.41)$ were observed. Regarding unipedal stance, the muscle pair did have an effect on the integral value of the anterior M-mode $\left(\chi_{2}^{2}=15.7\right.$; $p<0.001)$ and core muscles $\left(\chi_{2}^{2}=24.7 ; p<0.001\right)$. Pairwise comparisons revealed that the TA/VM integral was lower than VM/RA $(z=-3.95 ; p<0.001 ; r=-0.62)$, while the EO/ES integral was smaller than the RA/EO and RA/ES integrals $(z=4.9 ; p<0.001 ; r=0.77$ and $z=3.16$; $p=0.005 ; r=0.5$, respectively).

Comparisons between the integral values of both conditions showed several significant dissimilarities (Table 3). In the anterior muscle pairs, differences in TA/VM and TA/ RA coherence between the conditions were observed. The integral values were higher in the bipedal than unipedal stance. No differences were found between conditions in any posterior muscle pair. Regarding the core muscle pairs, $\mathrm{EO} / \mathrm{ES}$ coherence presented a higher integral value in the bipedal than in unipedal stance. Significant differences in mixed muscle pairs were observed. TA/BF and TA/ES revealed higher integral values in the bipedal than in unipedal stance. Finally, the coherence of TA/GM was higher in bipedal stance than in unipedal stance.

\section{Discussion}

In our study, we have found significant coherences in the $0-5 \mathrm{~Hz}$ frequency band in the anterior and posterior M-modes, core muscles and antagonist muscles of the leg during the balance tests. It was also observed that in the
Table 3 Integral coherence value of single pairs of muscles

\begin{tabular}{lll}
\hline & Bipedal stance & Unipedal stance \\
\hline TA/VM & $4.29(2.50)^{*}$ & $2.45(1.08)$ \\
TA/RA & $4.48(1.05)^{*}$ & $2.61(0.94)$ \\
VM/RA & $4.53(1.69)$ & $3.57(1.81)$ \\
GM/BF & $3.52(1.56)$ & $2.78(1.34)$ \\
GM/ES & $3.73(1.42)$ & $3.65(1.02)$ \\
BF/ES & $3.23(2.10)$ & $2.88(1.33)$ \\
RA/EO & $8.24(6.28)$ & $9.44(8.81)$ \\
RA/ES & $5.04(6.32)$ & $4.70(2.32)$ \\
EO/ES & $4.58(9.01)^{*}$ & $4.22(1.02)$ \\
GM/VM & $3.59(1.51)$ & $3.17(1.54)$ \\
GM/RA & $3.95(0.91)$ & $3.94(0.51)$ \\
VM/ES & $3.91(2.38)$ & $2.92(1.73)$ \\
TA/BF & $4.16(2.34)^{*}$ & $2.04(1.24)$ \\
TA/ES & $4.25(1.33)^{*}$ & $2.18(0.74)$ \\
BF/RA & $3.60(2.33)$ & $3.00(1.44)$ \\
TA-GM & $4.93(1.24)^{*}$ & $2.56(0.78)$ \\
VM-BF & $2.52(3.24)$ & $3.15(1.99)$ \\
\hline
\end{tabular}

Data are expressed as median (interquartile range)

$T A$ tibialis anterior, $G M$ gastrocnemius medialis, $V M$ vastus medialis, $B F$ biceps femoris, $R A$ rectus abdominis, $E O$ external oblique, $E S$ erector spinae

* Indicates significant differences between conditions $(p<0.05)$

groups formed by the anterior (not significant), posterior, core and antagonist muscles the coherence integral was greater than the mixed muscles in the bipedal condition (in theory not synergistic). This confirms the existence of common neural inputs to the muscles to create muscle synergies for maintaining balance, which has also been observed in the posterior and anterior muscles in other studies (DannaDos-Santos et al. 2014, 2015). However, up to date there have been no studies examining the core muscles during the static balance task. In addition, ours is the first study that analyses EMG coherence among the different muscle groups while performing single-leg static balance.

In the wide field of motor control and, particularly, in the field of static postural control, the study of motor variability derived from the redundant degrees of freedom and muscle actions is of great importance (Latash et al. 2002). The CNS should be able to develop appropriate strategies to solve problems, and these responses will be implemented by a system with multiple degrees of freedom. Some authors have proposed to simplify the problem by considering that the CNS organizes groups of structural elements or units with a common goal (Gelfand and Tsetlin 1966). In this way, the CNS seems to produce patterns of general operations that are sent to the structural units. If an error occurs in the execution of some of the elements that form a structural unit, other elements might compensate for 
the error. Systems operating according to these principles are considered to be synergies.

Considering the uncontrolled manifold (UCM) hypothesis, it was found that control of bipedal upright posture while performing voluntary sway or tasks that required arm movements was conducted by two muscular synergies (Krishnamoorthy et al. 2003) composed of an anterior and a posterior synergy. The anterior muscle synergy is responsible for moving the centre of mass forward, while the posterior acts in the opposite direction.

Although these synergies have been detected using the UCM hypothesis, it is still not known in depth how the CNS controls these structural units. Some authors suggest that it sends correlated neural inputs to the different muscles involved in each synergy, a hypothesis that has been checked in previous studies (Danna-Dos-Santos et al. 2014, 2015). In addition, it has been observed that when the sensorial visual inputs are removed, the correlated neural inputs diminish (Danna-Dos-Santos et al. 2015). Therefore, a deficit of visual information may imply that the CNS does not use the same muscular synergies, since these are task-specific synergies.

In the present study, we found that there are common neural inputs at low-frequency oscillations (i.e. between 0 and $5 \mathrm{~Hz}$ ) in the muscles forming the anterior and posterior $\mathrm{M}$-modes during the performance of static bipedal balance tasks. Previous studies delivered small differences in the frequency band in which significant coherence was found. For example, Danna-Dos-Santos et al. reported significant coherences between 0 and $20 \mathrm{~Hz}$ in the anterior M-mode in a "holding load forward" condition (Danna-Dos-Santos et al. 2014). However, the results of a recent paper show significant coherence only in the $0-10 \mathrm{~Hz}$ frequency band (Danna-Dos-Santos et al. 2015). Obata et al. compared the coherence integral in children, adults and the elderly, reporting significant coherence in the $0-4 \mathrm{~Hz}$ band in all three groups, as well as between 8 and $12 \mathrm{~Hz}$ in the older group (Obata et al. 2014). Only young adults were used in the present study, and our results are very similar to those obtained by Obata et al. (2014) regarding significant frequency bands.

This frequency band is related to a modulation known as the "common drive", which has a central origin (Kamen and De Luca 1992), but it is still not known in which centre it is generated. As it has been proven that people who suffered a cortical or capsular stroke keep the common drive (Farmer et al. 1993), these centres cannot be responsible for its generation. Oscillations in this frequency are produced in movements requiring constant or slowly increasing forces (Farmer et al. 1993; De Luca and Erim 1994). In this context, the fact that we found significant coherence values in the common drive frequency seems to be plausible, since it matches with the former description.
Although anterior and posterior muscles synergies were found by Krishnamoorthy et al. (2003), another study found more M-modes (without observing synergy) during the performance of different postural control tasks (Krishnamoorthy et al. 2004). It was hypothesized that the CNS chooses some M-modes from a task dependent "menu" of M-modes to maintain postural balance (Krishnamoorthy et al. 2004). In this study, reciprocal and co-contraction M-modes were found.

In this sense, our study reveals a large integral value of the spectral coherence in antagonist muscles and in core muscles. Therefore, this coherence could explain the M-modes co-contraction found in a previous study (Krishnamoorthy et al. 2004). Antagonist co-contraction has been considered efficient enough to increase joint stiffness and therefore to stabilize the joint during the performance of motor tasks such as upright posture (Hansen et al. 2002; Geertsen et al. 2013). Moreover, ankle joint stiffness has been accepted as one of the mechanisms involved in keeping balance in control studies (Baratto et al. 2002).

Regarding core muscles coherence, previous studies suggest that these muscles could provide the main muscle synergy for spine stability (Key 2013). In this regard, we found that the integral of the spectral coherence between these muscle groups was similar in both the anterior and posterior groups for the $0-5 \mathrm{~Hz}$ band. Furthermore, it is also possible that these muscles share neural inputs in other frequency bands, as we found values very close to the significance threshold in the rest of the analysed band width values.

However, it seems that the most plausible explanation is that the core muscles are compensating for inspiratory and expiratory movements that occur during breathing (Hodges et al. 2002; David et al. 2015). Although our study does not allow us to corroborate this hypothesis (i.e. we did not measure the respiratory rate in our subjects), it seems that the coherence we found between the different core muscles at lower frequencies points towards this type of CNS strategy to compensate for trunk movements due to breathing.

Of course, greater control of core muscles could also improve head stability, since these muscles control the movements of the lumbar vertebrae and the hips, which make a fundamental contribution to the width of movement in this body segment (Peterson et al. 2001).

Regarding the unipedal stance condition, it was found that the integral value was higher in posterior, core and antagonist muscles than in mixed and anterior muscles. This could mean that anterior synergies are not created during static unipedal balance, so that it would be interesting to use the UCM hypothesis to determine the actual muscles synergies in play during the single-leg stance task. Moreover, the integral value was lower in the unipedal than 
the bipedal stance condition in anterior (non-significant), posterior (significant) and core (non-significant) muscles, although the integral value for mixed and antagonist muscles was higher in the unipedal stance condition. This new landscape in EMG-EMG coherence during the unipedal stance test confirms that the synergies are task dependent (Latash et al. 2002).

Finally, stability and control parameters, such as the ellipse area covered and the mean velocity of the CoP, were significantly higher in unipedal stance. In addition, RMS EMG was larger in unipedal stance for the TA, GM, VM, EO and ES muscles. This increase in muscle activation (and therefore muscle force production) led to modifications in the common neural inputs of the different muscle pairs tested. This contradicts the results reported by Poston et al. during different force production in grasping tasks (Poston et al. 2010). However, not only force production but also muscle coordination could be different in unipedal and bipedal stance conditions.

Obviously, our work has some limitations that should be taken into account. Body segment position changes involved in balance control were not measured, since we could not perform kinematic analysis in our laboratory. This fact limits the conclusions of this study. Moreover, EMG recordings of both lower limbs during bipedal and unipedal stance tasks would provide additional information. This is important because for bipedal stance both lower limbs are working together, while the dominant limb acts alone in the unipedal stance test.

On the other hand, not having included a large number of trials restricted the type of analysis we could perform. Future research should include a larger number of trials for each condition in order to elucidate the synergies produced through analytic methods based on UCM hypothesis, such as principal component analysis (Krishnamoorthy et al. 2003). To corroborate our results, this type of experimental design should be carried out together with a high number of trials including the core muscles.

Even though the main findings of our study could be regarded as theoretical, the practical applications point out to the importance of adopting strategies involving core training for improving balance in persons with pathologies entailing problems in balance control (Park and Hwangbo 2014).

Our main conclusion is that the core and antagonist muscle groups, such as the anterior and posterior muscles, share low-frequency neural inputs $(0-5 \mathrm{~Hz})$. These common inputs might be responsible of the assembly of the M-modes. In this sense, our results support the hypothesis that the CNS is dictating the M-modes conformation and therefore responsible for postural control handling with the additional problem of high degrees of freedom. However, our results do not permit us to identify exactly where the process takes place: either at the supraspinal or at spinal medulla levels of the nervous system. In addition, we found important differences between the unipedal and bipedal stance conditions regarding postural control and stability, as well as in muscle activation. It is thus possible that the muscle synergies involved in unipedal stance tasks are different to those required for the bipedal stance condition.

Acknowledgments Open access funding provided by University of Natural Resources and Life Sciences Vienna (BOKU). This study has been supported by two research grants of the University of Valencia, Spain (Ayudas para estancias de investigación: UV-INV_EPDI15272601 y UV-INV EPDI15-276152).

\section{Compliance with ethical standards}

Ethical approval All procedures performed in studies involving human participants were in accordance with the ethical standards of the institutional and/or national research committee and with the 1964 Declaration of Helsinki and its later amendments or comparable ethical standards.

Open Access This article is distributed under the terms of the Creative Commons Attribution 4.0 International License (http://creativecommons.org/licenses/by/4.0/), which permits unrestricted use, distribution, and reproduction in any medium, provided you give appropriate credit to the original author(s) and the source, provide a link to the Creative Commons license, and indicate if changes were made.

\section{References}

Asai Y, Tasaka Y, Nomura K et al (2009) A model of postural control in quiet standing: robust compensation of delay-induced instability using intermittent activation of feedback control. PLoS One 4:e6169. doi:10.1371/journal.pone.0006169

Baratto L, Morasso PG, Re C, Spada G (2002) A new look at posturographic analysis in the clinical context: sway-density versus other parameterization techniques. Mot Control 6:246-270

Bisson EJ, McEwen D, Lajoie Y, Bilodeau M (2011) Effects of ankle and hip muscle fatigue on postural sway and attentional demands during unipedal stance. Gait Posture 33:83-87. doi:10.1016/j. gaitpost.2010.10.001

Bizid R, Margnes E, François Y et al (2009) Effects of knee and ankle muscle fatigue on postural control in the unipedal stance. Eur $\mathrm{J}$ Appl Physiol 106:375-380. doi:10.1007/s00421-009-1029-2

Boonstra TW, Breakspear M (2012) Neural mechanisms of intermuscular coherence: implications for the rectification of surface electromyography. J Neurophysiol 107:796-807. doi:10.1152/ jn.00066.2011

Danna-Dos-Santos A, Degani AM, Latash ML (2008) Flexible muscle modes and synergies in challenging whole-body tasks. Exp Brain Res 189:171-187. doi:10.1007/s00221-008-1413-x

Danna-Dos-Santos A, Boonstra TW, Degani AM et al (2014) Multi-muscle control during bipedal stance: an EMG-EMG analysis approach. Exp Brain Res 232:75-87. doi:10.1007/ s00221-013-3721-z

Danna-Dos-Santos A, Degani AM, Boonstra TW et al (2015) The influence of visual information on multi-muscle control during quiet stance: a spectral analysis approach. Exp Brain Res 233:657-669. doi:10.1007/s00221-014-4145-0

David P, Terrien J, Petitjean M (2015) Postural- and respiratoryrelated activities of abdominal muscles during post-exercise 
hyperventilation. Gait Posture 41:899-904. doi:10.1016/j. gaitpost.2015.03.012

De Luca CJ, Erim Z (1994) Common drive of motor units in regulation of muscle force. Trends Neurosci 17:299-305

Farina D, Negro F, Jiang N (2013) Identification of common synaptic inputs to motor neurons from the rectified electromyogram. J Physiol 591:2403-2418. doi:10.1113/jphysiol.2012.246082

Farmer S, Swash M, Ingram D, Stephens JA (1993) Changes in motor unit synchronization following central nervous lesions in man. $\mathrm{J}$ Physiol 463:83-105

Gawthrop P, Loram I, Lakie M, Gollee H (2011) Intermittent control: a computational theory of human control. Biol Cybern 104:3151. doi:10.1007/s00422-010-0416-4

Geertsen SS, Kjær M, Pedersen KK et al (2013) Central common drive to antagonistic ankle muscles in relation to short-term cocontraction training in nondancers and professional ballet dancers. J Appl Physiol Bethesda Md 1985 115:1075-1081. doi:10.1152/japplphysiol.00707.2012

Gelfand I, Tsetlin ML (1966) On mathematical modeling of the mechanisms of the central nervous system. In: Gelfand IM, Gurfinkel VS, Fomin SV, Tsetlin ML (eds) Models of the structural -functional organization of certain biological systems. Nauka, Moscow, pp 9-26

Grosse P, Cassidy MJ, Brown P (2002) EEG-EMG, MEG-EMG and EMG-EMG frequency analysis: physiological principles and clinical applications. Clin Neurophysiol 113:1523-1531. doi:10.1016/S1388-2457(02)00223-7

Günther M, Grimmer S, Siebert T, Blickhan R (2009) All leg joints contribute to quiet human stance: a mechanical analysis. J Biomech 42:2739-2746. doi:10.1016/j.jbiomech.2009.08.014

Günther M, Putsche P, Leistritz L, Grimmer S (2011) Phase synchronisation of the three leg joints in quiet human stance. Gait Posture 33:412-417. doi:10.1016/j.gaitpost.2010.12.014

Hansen S, Hansen NL, Christensen LOD et al (2002) Coupling of antagonistic ankle muscles during co-contraction in humans. Exp Brain Res 146:282-292. doi:10.1007/s00221-002-1152-3

Hertel J, Gay MR, Denegar CR (2002) Differences in postural control during single-leg stance among healthy individuals with different foot types. J Athl Train 37:129-132

Hodges PW, Gurfinkel VS, Brumagne S et al (2002) Coexistence of stability and mobility in postural control: evidence from postural compensation for respiration. Exp Brain Res 144:293-302. doi:10.1007/s00221-002-1040-x

Kamen G, De Luca CJ (1992) Firing rate interactions among human orbicularis oris motor units. Int J Neurosci 64:167-175

Key J (2013) "The core": understanding it, and retraining its dysfunction. J Bodyw Mov Ther 17:541-559. doi:10.1016/j. jbmt.2013.03.012

Krishnamoorthy V, Latash ML, Scholz JP, Zatsiorsky VM (2003) Muscle synergies during shifts of the center of pressure by standing persons. Exp Brain Res 152:281-292. doi:10.1007/ s00221-003-1574-6

Krishnamoorthy V, Latash ML, Scholz JP, Zatsiorsky VM (2004) Muscle modes during shifts of the center of pressure by standing persons: effect of instability and additional support. Exp Brain Res 157:18-31. doi:10.1007/s00221-003-1812-y

Latash ML, Scholz JP, Schöner G (2002) Motor control strategies revealed in the structure of motor variability. Exerc Sport Sci Rev 30:26-31

Mak JNF, Hu Y, Luk KDK (2010) An automated ECG-artifact removal method for trunk muscle surface EMG recordings. Med Eng Phys 32:840-848. doi:10.1016/j.medengphy.2010.05.007

Masani K, Popovic MR, Nakazawa K et al (2003) Importance of body sway velocity information in controlling ankle extensor activities during quiet stance. J Neurophysiol 90:3774-3782. doi:10.1152/ jn.00730.2002

Obata H, Abe MO, Masani K, Nakazawa K (2014) Modulation between bilateral legs and within unilateral muscle synergists of postural muscle activity changes with development and aging. Exp Brain Res 232:1-11. doi:10.1007/s00221-013-3702-2

Paillard T, Noé F, Rivière T et al (2006) Postural performance and strategy in the unipedal stance of soccer players at different levels of competition. J Athl Train 41:172-176

Park JH, Hwangbo G (2014) The effect of trunk stabilization exercises using a sling on the balance of patients with hemiplegia. J Phys Ther Sci 26:219-221. doi:10.1589/jpts.26.219

Peterson BW, Choi H, Hain T et al (2001) Dynamic and kinematic strategies for head movement control. Ann NY Acad Sci 942:381-393

Poston B, Danna-Dos Santos A, Jesunathadas M et al (2010) Forceindependent distribution of correlated neural inputs to hand muscles during three-digit grasping. J Neurophysiol 104:1141-1154. doi:10.1152/jn.00185.2010

Rosenberg JR, Amjad AM, Breeze P et al (1989) The Fourier approach to the identification of functional coupling between neuronal spike trains. Prog Biophys Mol Biol 53:1-31

Shumway-Cook A, Woollacott M (2007) Motor control. Translating research into clinical practice. Lippincott Williams \& Wilkins, Philadelphia

Suzuki Y, Nomura T, Casadio M, Morasso P (2012) Intermittent control with ankle, hip, and mixed strategies during quiet standing: a theoretical proposal based on a double inverted pendulum model. J Theor Biol 310:55-79. doi:10.1016/j.jtbi.2012.06.019

Vuillerme N, Danion F, Marin L et al (2001) The effect of expertise in gymnastics on postural control. Neurosci Lett 303:83-86

Ward NJ, Farmer SF, Berthouze L, Halliday DM (2013) Rectification of EMG in low force contractions improves detection of motor unit coherence in the beta-frequency band. J Neurophysiol 110:1744-1750. doi:10.1152/jn.00296.2013 